Georgian Mathematical Journal

Volume 8 (2001), Number 4, 713-726

\title{
GLOBAL GEOMETRIC ASPECTS OF RIEMANN-HILBERT PROBLEMS
}

\author{
B. BOJARSKI AND G. KHIMSHIASHVILI
}

Dedicated to N. N. Vakhania

on the occasion of his 70th birthday

\begin{abstract}
We discuss some global properties of an abstract geometric model for Riemann-Hilbert problems introduced by the first author. In particular, we compute the homotopy groups of elliptic Riemann-Hilbert problems and describe some connections with the theory of Fredholm structures which enable one to introduce more subtle geometrical and topological invariants for families of such problems.
\end{abstract}

2000 Mathematics Subject Classification: 58G03, 59G20.

Key words and phrases: Riemann-Hilbert transmission problem, Fredholm pair of subspaces, Hilbert-Schmidt operator, Fredholm Grassmanian of a polarized Hilbert space, Hilbert manifold, Fredholm structure.

\section{INTRODUCTION}

As is well known, the classical Riemann-Hilbert (transmission) problem has deep and far reaching connections with many important problems in analysis and geometry (see, e.g., [21], [1], [2], [3]). Thus in addition to a comprehensive analytic theory [21], it also has some natural global geometric aspects. In particular as was suggested in [2] (see also [3]) the totality of elliptic Riemann-Hilbert problems permits a visual geometric description in terms of Fredholm pairs of subspaces of an appropriate functional space. This interpretation enables one to study various global aspects of the Riemann-Hilbert problem in an abstract setting, which eventually led to some conceptual developments [4], [5], [15], [16] and non-trivial geometric results about the so-called Fredholm Grassmanian [2], [4], [17], [18], [24]. Closely related concepts and constructions appeared useful in the geometric theory of loop groups of compact Lie groups [22], [16]. The goal of this paper is to present a coherent exposition of those geometric results and discuss some new developments in the same direction.

In particular, we describe the homotopy type of Fredholm Grassmanians, show that they can be endowed with smooth manifold structures and explain how one can put them in the context of Fredholm structures. We also indicate some perspectives steming from such an approach and formulate several seemingly interesting problems.

ISSN 1072-947X / \$8.00 / C) Heldermann Verlag www.heldermann.de 


\section{The Riemann-Hilbert Transmission Problem}

The classical formulation of the Riemann-Hilbert problem is related to the decomposition of the extended complex plane $\overline{\mathbf{C}}$ (Riemann sphere) into two complementary domains with a smooth common boundary $\Gamma$. In the simplest case

$$
\overline{\mathbf{C}}=D_{+} \cup T \cup D_{-},
$$

where $D_{+}$is the unit disc, $T$ stands for the unit circle, and $D_{-}$is the complementary domain containing the infinite point $\infty$ (the North Pole of Riemann sphere). Let $A\left(D_{ \pm}\right)=C\left(\bar{D}_{ \pm}\right) \cap H\left(D_{ \pm}\right)$denote the set of all complex valued (vector) functions which are continuous in the closure of the corresponding domain and holomorphic inside.

The problem, known as Riemann-Hilbert transmission problem or linear conjugation problem, is to describe the totality of piecewise holomorphic (vector) functions $\left(X_{+}, X_{-}\right) \in A\left(D_{+}\right) \times A\left(D_{-}\right)$with the normalizing condition $X_{-}(\infty)=0$, whose boundary values satisfy the transmission condition

$$
X_{+}(t)=G(t) X_{-}(t)+h(t), t \in T,
$$

where $h(t)$ is a given (vector) function and $G(t)$ is a (matrix) function of the corresponding size.

The same problem can be of course formulated on any Riemann surface but we stick here to the zero genus case (Riemann sphere) as above. Solutions may be considered in various functional spaces. For example, the problem can be placed in a Hilbert space context by working with square-integrable functions and this is well-suited for studying global geometric aspects of the problem.

Solvability and other properties of this problem are very well understood (see, e.g., [21]). For example, the problem is Fredholm if the coefficient matrix $G(t)$ is non-degenerate at every point of the unit circle and belongs to some Hölder class. The index of this problem appears to be equal to the winding number (topological degree) of the determinant $\operatorname{det} G(t)$, i.e., it is equal to the divided by $2 \pi$ increment of the argument of $\operatorname{det} G(t)$ along the unit circle [21]. One can also express the kernel and cokernel dimension in terms of the socalled partial indices of the matrix function $G(t)$ which are defined in terms of Birkhoff factorization of non-degenerate matrix functions on the circle [21], [1]. The partial indices exhibit quite non-trivial behaviour closely related to the properties of holomorphic vector bundles over the Riemann sphere [1], [3].

It turns out that it is also possible to describe some global properties of the set of all such problems, which will be our main concern in the sequel. We begin by recalling an abstract geometric model for this set suggested in [2]. For simplicity and brevity we work in the framework of Hilbert spaces. However most of our constructions and results apparently remain valid for a wide class of Banach spaces. They can be also generalized in the context of Hilbert modules over $C^{*}$-algebras [18]. 


\section{Fredholm Pairs and Grassmanians}

Let $H$ be a complex Hilbert space and $M, N$ be its closed infinite-dimensional subspaces. A pair $\mathbf{P}=(M, N)$ is called a Fredholm pair (FP) if $M+N$ is a closed subspace of finite codimension $b_{\mathbf{P}}$, and $\operatorname{dim}(M \cap N)=a_{\mathbf{P}}$ is also finite. In such a case the difference $a_{\mathbf{P}}-b_{\mathbf{P}}=i(M, N)$ is called the index of Fredholm pair $\mathbf{P}$.

The concept of Fredholm pair was introduced in 60-ties by T.Kato [14] who established in particular that such pairs and their indices are stable with respect to continuous deformations of the subspaces in question. For a precise formulation of this property see [14] or [2].

In order to characterize Fredholm pairs, certain classes of bounded linear operators in $H$ were introduced in [2]. Let $L(H)$ denote the algebra of bounded linear operators in $H$ and $G L(H)$ denote the group of operators possessing a bounded inverse. Let $J$ be a fixed two-sided ideal in $L(H)$. For example, one can take the (unique closed two-sided) ideal $K$ of compact (completely continuous) operators or the subideal $K_{0}$ consisting of finite rank operators.

For a given operator $S \in L(H)$, let $C(S, J)$ denote the subalgebra of operators $A \in L(H)$ such that the commutator $[A, S]=A S-S A$ belongs to the ideal $J$. The intersection $C(S, J) \cap G L(H)$ will be denoted by $G L(S, J)$, clearly it is a subgroup of $G L(H)$ (not necessarily a closed one).

As was explained in [2] the classical singular integral operators and linear conjugation problems can be interpreted as elements of the algebra $C(P, K)$, where $P$ is an orthogonal projector with infinite-dimensional image and kernel. Many topological properties of such operators and related Grassmanians remain valid if one changes the ideal $K$ by certain subideal $J$ as above.

Definition 1 ([2]). Let $P$ be an orthogonal projection on a closed subspace in $H$ such that $\operatorname{dim} \operatorname{im} P=\operatorname{dim} \operatorname{ker} P=\infty$. The algebra $C(P, J)$ is called the algebra of abstract singular operators associated with an ideal $J, K_{0} \subset J \subset K$.

In the sequel we will be mainly interested in the group of invertible (abstract) singular operators $G L(P, J)$. If $M$ is a closed linear subspace of $H$ and $A \in$ $G L(H)$ an invertible operator in $H$, then $A(M)$ denotes the image of $M$ under $A$ and we think of it as a subspace $M$ rotated by $A$. Let $P_{M}$ denote any projection onto $M$, i.e., the range of $P_{M}$ is $M$ and $\left(P_{M}\right)^{2}=P_{M}$. Of course there exist many projectors with the given range $M$ but, in a Hilbert space, the condition that $P_{M}$ is self-adjoint (or orthogonal) specifies it in a unique way. We now introduce the complementary projections $P=P_{M}, Q=I d-P$ and present a very precise characterization of Fredholm pairs which was obtained in [2].

Theorem 1 ([2]). A pair $(M, N)$ of closed subspaces of a Hilbert space is a Fredholm pair if and only if it has the form $\left(M, A\left(M^{\perp}\right)\right)$ for some operator $A \in G(P, K)$. The operator $\Phi \in L(H)$ defined by the formula

$$
\Phi(x)=P x+A Q x
$$


is a Fredholm operator with ind $\Phi=i(M, N)$. Any operator of this form in $L(H)$ is a Fredholm operator.

It turns out that in many problems it becomes necessary to consider the set of all Fredholm pairs with a fixed first subspace. In other words, one chooses a closed infinite-dimensional and infinite-codimensional subspace $M$ and considers the so-called Fredholm Grassmanian consisting of all subspaces $N$ such that $(M, N)$ is a Fredholm pair (cf. [22], Ch.7). This is actually a "leaf" in the Grassmanian of all Fredholm pairs and one may represent the whole Grassmanian as a fibration with a fiber isomorphic to this leaf.

This definition permits several useful modifications which we present following [22]. Consider a complex Hilbert space decomposed in an orthogonal direct sum $H=H_{+} \oplus H_{-}$and choose a positive number $s$. For further use we need a family of subideals in $K(H)$ which is defined as follows (cf. [13]).

Recall that for any bounded operator $A \in L(H)$ the product $A^{*} A$ is a nonnegative self-adjoint operator, so it has a well-defined square root $|A|=\left(A^{*} A\right)^{1 / 2}$ (see, e.g., [23]). If $A$ is compact, then $A^{*} A$ is also compact and $|A|$ has a discrete sequence of eigenvalues

$$
\mu_{1}(A) \geq \mu_{2}(A) \geq \ldots
$$

tending to zero. The $\mu_{n}(A)$ are called singular values of $A$. For a finite $s \geq 1$ one can consider the expression (sth norm of $A$ )

$$
\|A\|_{s}=\left[\sum_{j=1}^{\infty}\left(\mu_{j}(A)\right)^{s}\right]^{1 / s}
$$

and define the $s$ th Schatten ideal $K_{s}$ as the collection of all compact operators $A$ with a finite $s$ th norm (s-summable operators) [23].

Using elementary inequalities it is easy to check that $K_{s}$ is really a twosided ideal in $L(H)$. These ideals are not closed in $L(H)$ with its usual norm topology but if one endows $K_{s}$ with the $s$ th norm as above then $K_{s}$ becomes a Banach space [23]. Two special cases are well-known: $K_{1}$ is the ideal of trace class operators and $K_{2}$ is the ideal of Hilbert-Schmidt operators. For $s=2$, the above norm is called the Hilbert-Schmidt norm of $A$ and it is well known that $K_{2}(H)$ endowed with this norm becomes a Hilbert space (see, e.g., [23]). Obviously $K_{1} \subset K_{s} \subset K_{r}$ for $1<s<r$ so one obtains a chain of ideals starting with $K_{1}$. For convenience we set $K_{\infty}=K$ and obtain an increasing chain of ideals $K_{s}$ with $s \in[1, \infty]$.

Of course one can introduce similar definitions for a linear operator $A$ acting between two different Hilbert spaces, e.g., for an operator from one subspace $M$ to another subspace $N$ of a fixed Hilbert space $H$. In particular we can consider the classes $K_{s}\left(H_{ \pm}, H_{\mp}\right)$. Let us also denote by $F(M, N)$ the space of all Fredholm operators from $M$ to $N$.

Definition 2 ([22]). The sth Fredholm Grassmanian of a polarized Hilbert 
space $H$ is defined as

$$
\begin{aligned}
G r_{F}^{s}(H)=\{W \subset H: & \pi_{+} \mid W \text { is an operator from } F\left(W, H_{+}\right), \\
& \left.\pi_{-} \mid W \text { is an operator from } K_{s}\left(W, H_{-}\right)\right\} .
\end{aligned}
$$

These Grassmanians are of the major interest for us. Actually, many of their topological properties (e.g., the homotopy type discussed in the next section) do not depend on the number $s$ appearing in the definition. On the other hand, more subtle properties like manifold structures and characteristic classes of $G r_{F}^{s}$ do depend on $s$ in a quite essential way. As follows from the discussion in [12] this is a delicate issue and we circumvent it by properly choosing the context.

As follows from the results of [22], it is especially convenient to work with the Grassmanian $G r_{F}^{2}(H)$ defined by the condition that the second projection $\pi_{-}$restricted to $W$ is a Hilbert-Schmidt operator. Following [22] we denote it by $G r_{r}(H)$ and call the restricted Grassmanian of $H$.

Fredholm Grassmanians appear to have interesting analytic and topological properties. It turns out that Grassmanian $G r_{F}^{s}$ can be turned into Banach manifolds modelled on Schatten ideal $K_{s}$. In particular $G r_{r}(H)$ has a natural structure of a Hilbert manifold modelled on the Hilbert space $K_{2}(H)$ [22]. All these Grassmanians have the same homotopy type (see Theorem 2 below). Moreover certain natural subsets of Grassmanians $G r_{F}^{s}$ can be endowed with socalled Fredholm structures [9], which suggests in particular that one can define various global topological invariants of $G r_{F}^{s}(H)$.

Definition 1 also yields a family of subgroups $G L^{s}=G L\left(\pi_{+}, K_{s}\right)$ of $G L\left(\pi_{+}, K\right)$ $(s \geq 1)$. For our purposes especially important is the subgroup $G L\left(\pi_{+}, K_{2}\right)$ which naturally acts on $G r_{r}(H)$.

Definition 3 ([22]). The restricted linear group $G L_{r}(H)$ is defined as the subgroup of $G L\left(\pi_{+}, K\right)$ consisting of all operators $A$ such that the commutator $\left[A, \pi_{+}\right]$belongs to the Hilbert-Schmidt class $K_{2}(H)$.

From the very definition it follows that $G L^{s}$ acts on $G r^{s}$ and by merely an examination of the proof of Theorem 1 given in [2] (cf. also [22], Ch.7) one finds out that these actions are transitive. In order to give the most convenient description of the isotropy subgroups of these actions, we follow the presentation of [22] and introduce a subgroup $U^{s}(H)=U(H) \cap G L^{s}(H)$ consisting of all unitary operators from $G L^{s}$. For $s=2$ this subgroup is denoted by $U_{r}$. Now the description of isotropy groups is available by the same way of reasoning which was applied in [22] for $s=2$.

Proposition 1. The subgroup $U^{s}(H)$ acts transitively on $\operatorname{Gr}^{s}(H)$ and the isotropy subgroup of the subspace $H_{+}$is isomorphic to $U\left(H_{+}\right) \times U\left(H_{-}\right)$.

From the existence of a polar decomposition for a bounded operator on $H$ it follows that subgroup $U^{s}(H)$ is a retract of $G L^{s}$ and it is straightforward to obtain similar conclusions for the actions of $G L^{s}$. 
Corollary 1. The group $G L^{s}$ acts transitively on the Grassmanian $G r^{s}(H)$ and the isotropy groups of this action are contractible.

Thus such an action obviously defines a fibration with contractible fibers and it is well known that for such fibrations the total space $\left(G L^{s}\right)$ and the base $\left(G r^{s}\right)$ are homotopy equivalent [9].

Corollary 2. For any $s \geq 1$, the Grassmanian $G r^{s}$ and the group $G L^{s}$ have the same homotopy type. In particular, $G L_{r}$ is homotopy equivalent to $G r_{r}$.

Remark 1. As we will see in the next section, all the groups $G L\left(\pi_{+}, J\right)$ have the same homotopy type for any ideal $J$ between $K_{0}$ and $K$. In particular, this is true for every Schatten ideal $K_{s}$. Thus all the above groups and Grassmanians have the same homotopy type.

We are now ready to have a closer look at the topology of $G r^{s}$ and $G L^{s}$ which will be our main concern in the next section.

\section{The Topology of Fredholm Grassmanians}

The homotopy type of $G L_{r}$ and $G r_{r}$ is described in the following statement which was obtained in [16] and [24]. This gives an answer to a question posed in [2].

Theorem 2. For any $s \in[1, \infty]$, the homotopy groups of the group $G L^{s}$ and Fredholm Grassmanian $\mathrm{Gr}^{s}$ are given by the formulae

$$
\pi_{0} \cong \mathbf{Z} ; \quad \pi_{2 k+1} \cong \mathbf{Z}, \pi_{2 k+2}=0, k \geq 0 .
$$

Proof. In virtue of Corollary 2, it is sufficient to determine the homotopy type of $G L^{s}$ which we denote simply by $G$. To this end let us consider a certain fibration

$$
p_{1}: G L^{s} \rightarrow F\left(H_{+}, H_{+}\right) \times K_{s}\left(H_{+}, H_{-}\right)
$$

defined in the following way.

Write any element (operator) $A \in G L^{s}$ as a $(2 \times 2)$-matrix of operators

$$
\left(\begin{array}{ll}
a & b \\
c & d
\end{array}\right)
$$

corresponding to the given polarization of $H$ (thus $a$ is a bounded operator from to $H_{+}$to $H_{+}$and so on).

Then define $p_{1}(A)$ as the first column of this matrix, i.e., $p_{1}(A)=(a, c)$. It is evident that the image $X=I m p_{1}$ is an open subset of the target space. Introduce now a subgroup $G_{1} \subset G$ of elements of $G$ defined by upper-triangular matrices of the form

$$
\left(\begin{array}{cc}
I_{+} & b \\
0 & d
\end{array}\right)
$$

where $I_{+}$denotes the identity operator on $H_{+}$.

Lemma 1. The subgroup $G_{1}$ is contractible. 
Indeed, notice first that in this representation the operator $d$ is always invertible, in other words the set of possible $d$-s appearing in the last formula is exactly $G L\left(H_{-}\right)$. As to $b$ it can be an arbitrary operator from $K_{s}\left(H_{-}, H_{+}\right)$. Thus the subgroup $G_{1}$ as a topological space is homeomorphic to the product $G L\left(H_{-}\right) \times K_{s}\left(H_{-}, H_{+}\right)$. By Kuiper's theorem [19], the first factor is contractible and the second factor, being a vector space, is also contractible. Thus we conclude that $G_{1}$ is contractible.

Now it is straightforward to verify our next claim.

Lemma 2. $p_{1}(A)=p_{1}\left(A^{\prime}\right)$ if and only if there exists a $T \in G_{1}$ such that $A=A^{\prime} T$.

Thus we conclude that $X$ is the homogeneous space $G / G_{1}$ which is apparently a fibration with the fibers isomorphic to $G_{1}$. As was already explained in the previous section, this implies that $G$ is homotopy equivalent to $X$.

Consider now the mapping $\pi_{1}: X \rightarrow F\left(H_{+}, H_{+}\right)$defined as the restriction of the first projection, i.e., $\pi_{1}(a, c)=a$. We want to show that this is also a surjective mapping with contractible fibers. Then, by the same reasoning as above, we will be able to conclude that $G$ is homotopy equivalent to $F\left(H_{+}, H_{+}\right)$. Since it is well known that the homotopy groups of the latter space are exactly those as were given in the statement of the theorem, this would complete the proof.

Thus we see that it remains to verify next two lemmas.

Lemma 3. Each $a \in F\left(H_{+}, H_{+}\right)$can appear as an left-upper element of a two-by-two matrix above.

Lemma 4. For each $a \in F\left(H_{+}, H_{+}\right)$, the set of all $c$ such that $(a, c)^{*}$ can appear as the first column of a matrix representing an element of $G$, coincides with the set of all $c \in K_{s}\left(H_{+}, H_{-}\right)$such that $c \mid$ ker $a$ is injective. The set of all such $c$ is a contractible subset in $K_{s}\left(H_{+}, H_{-}\right)$.

The first of these two lemmas follows from a well-known procedure of regularizing of a Fredholm operator. One takes any embedding $c$ of ker $a$ into $H_{-}$and takes $b$ to be a finite rank operator from $H_{-}$onto $(i m a)^{\perp}$. Then one can obtain an appropriate $d$ by taking any epimorphism of $H_{-}$onto ker $b$ with the kernel $i m c$. It is trivial to check that this really defines an operator from $G L\left(\pi_{+}, K_{0}\right)$ so this construction does the job simultaneously for all ideals $K_{s}$ with $s \geq 1$ and the first lemma is proved.

Moreover, from this argument it becomes evident that the only restriction on $c$ in order that it could "accompany" a given $a$ in $G L^{s}$ is that it maps ker $a$ injectively into $H_{-}$(again no matter which ideal $K_{s}$ is considered). On the other hand if $c$ appears as the lower-left corner element of such a matrix then its kernel should be trivial.

The last statement of the last lemma follows from the fact that the set of all such $c$ is apparently homeomorphic to the set of all $n$-tuples of linearly independent vectors (i.e., $n$-frames) in $H_{-}$, where $n=\operatorname{dim} \operatorname{ker} a$. As is well known all 
spaces of frames are contractible [9] so we obtain the desired conclusion. This completes the proof of the theorem.

As was shown in [22], the restricted Grassmanian has also a remarkable structure of a cellular complex (CW-complex) which is closely related to the so-called partial indices [1] and gives a visual interpretation of certain phenomena discussed in [1], [2]. Moreover, Fredholm Grassmanians can be turned into differentiable manifolds, which enables one to construct an analogue of the Morse theory and recover in this way the cellular structure obtained from the partial indices [22], [17]. We describe here a simple explicit way of introducing differentiable manifold structures on Fredholm Grassmanians $G r^{s}$.

Theorem 3. For any finite $s \geq 1$, the Grassmanian $G r^{s}(H)$ is a differentiable manifold modelled on Banach space $K_{s}(H)$.

Proof. We first construct a natural atlas on $G r^{s}$ (cf. [22] for $s=2$ ). Notice that the graph of every $s$-summable operator $w: H_{+} \rightarrow H_{-}$belongs to $G r^{s}$. Since the sum of a Fredholm operator and an $s$-summable operator is a Fredholm operator, one concludes that, for every $W \in G r^{s}$, the graph of any $s$-summable operator from $W$ to $W^{\perp}$ also belongs to $G r^{s}$. Such graphs constitute an open subset $U_{W} \in G r_{r}$ consisting of all $W^{\prime}$ such that the orthogonal projection $W^{\prime} \rightarrow W$ is an isomorphism. Obviously this open subset is in a one-to-one correspondence with the space $K_{s}\left(W, W^{\perp}\right)$ of $s$-summable operators from $W$ to $W^{\perp}$, which defines an atlas on $G r^{s}$.

We now describe an explicit form of the transition diffeomorphisms of this atlas and verify that this atlas really defines a structure of a differentiable manifold, i.e., differentials $D\left(g_{i} \circ g_{j}^{-1}\right)(p)$ are bounded operators in $K_{s}(H)$. This would apparently complete the proof.

Let $U_{V}$ and $U_{W}$ be the open sets in $G r^{s}$ corresponding to the spaces $H_{1}=$ $K_{s}\left(V, V^{\perp}\right)$ and $H_{2}=K_{s}\left(W, W^{\perp}\right)$. Let us show that the images $H_{12}$ and $H_{21}$ of the intersection $U_{V} \cap U_{W}$ in these spaces are open and the corresponding "change of coordinates" $H_{12} \rightarrow H_{21}$ is continuously differentiable.

Let us consider the identity transformation of $H$ as an operator

$$
V \oplus V^{\perp} \rightarrow W \oplus W^{\perp}
$$

and write it in the form of a two-by-two matrix of operators

$$
\left(\begin{array}{ll}
a & b \\
c & d
\end{array}\right)
$$

corresponding to these direct sum decompositions. Here $a$ is an operator from $V$ to $W$, and so on (cf. the proof of Theorem 2).

From the fact that both $V$ and $W$ belong to $G r^{s}$ it follows easily that the diagonal terms $a, d$ are Fredholm operators while $b$ and $c$ are operators of $K_{s}$ class. Suppose now that a subspace $L \in U_{V} \cap U_{W}$ is simultaneously the graph 
of operators $T_{1}: V \rightarrow V^{\perp}$ and $T_{2}: W \rightarrow W^{\perp}$. Then operators

$$
\left(\begin{array}{ll}
a & b \\
c & d
\end{array}\right)\left(\begin{array}{c}
1 \\
T_{1}
\end{array}\right) \text { and }\left(\begin{array}{c}
1 \\
T_{2}
\end{array}\right) q
$$

should coincide as operators from $V$ to $W \oplus W^{\perp}$ for some isomorphism $q: V \rightarrow$ $W$. This implies that

$$
T_{2}=\left(c+d T_{1}\right)\left(a+b T_{1}\right)^{-1} .
$$

The last relation apparently shows that $T_{2}$ is a continuous function of $T_{1}$ on the open set $H_{12}=\left\{T_{1} \in H_{1}: a+b T_{1}\right.$ is invertible $\}$.

This means that the atlas $U_{W}$ really defines on $G r^{s}$ a structure of a topological manifold and it remains to verify that the differentials of coordinate changes in this atlas do exist and they are bounded linear operators as operators in $K_{s}(H)$. To this end let us compute the differential of $T_{2}$ as a function of $T_{1}$. By a standard application of Leibniz rule for operator-valued functions one obtains:

$$
D T_{2}\left(T_{1}\right)=d\left(a+b T_{1}\right)^{-1}-\left(c+d T_{1}\right)\left(a+b T_{1}\right) b\left(a+b T_{1}\right)^{-1} .
$$

Now one can make a straightforward examination the linear operator in $K_{s}(H)$ defined as the multiplication by the right hand side of this formula, using the Neumann series for the inverse $\left(a+b T_{1}\right)^{-1}$, and verify that it defines a bounded linear operator on $K_{s}(H)$. Thus this atlas really defines a differentiable manifold structure on $G r^{s}$ and the proof is complete.

Remark 2. In the case $s=2$ the same atlas defines a holomorphic Hilbert manifold structure on $G r_{r}$ (modelled on the Hilbert space $K_{2}(H)$ with its Hilbert-Schmidt norm) (cf. [22]).

Remark 3. Apparently one can introduce similar operator groups and Grassmanians in any Banach space. The above results remain valid for wide classes of separable Banach spaces with basis and contractible general linear group but here we cannot dwell upon that issue.

\section{Loop Groups and Fredholm Structures}

In order to obtain a proper framework for discussing more subtle geometric properties of Fredholm Grassmanians we proceed by describing some connections with the Fredholm structures theory [10]. As was observed in [16], [17], certain dense subsets of these Grassmanians can be endowed with Fredholm structures. This fact seems to be quite remarkable since a Fredholm structure on an infinite-dimensional manifold enables one to introduce non-trivial global geometric and topological invariants of this manifold. The reason for this circumstance is that Fredholm Grassmanians are closely related to loop groups of compact Lie groups [22] and such loop groups can be endowed with some natural Fredholm structures [15], [16], [12]. Our discussion of this issue is based on the results of [16] and [12] but we present them with a view to Fredholm Grassmanians. 
For simplicity we only consider the classical case corresponding to the loop group of unitary group $U_{n}$. Recall that Riemann-Hilbert problems for arbitrary compact Lie groups were studied in [16]. The discussion below is applicable for arbitrary compact Lie groups.

Recall that a Fredholm structure on an (infinite-dimensional) Banach manifold $M$ modelled on a Banach space $E$ is defined as by an atlas $\left(U_{i}, g_{i}\right)$ on $M$ such that for any point $p \in g_{j}\left(U_{i} \cap U_{j}\right)$ the differential (Fréchet derivative) of the transition diffeomorphism $D\left(g_{i} \circ g_{j}^{-1}\right)(p)$ is an invertible operator of the form "identity + compact" [9].

Existence of a Fredholm structure on an infinite-dimensional Banach manifold is a rare event and such manifolds possess various interesting global geometric and topological invariants (curvatures, characteristic classes) [10]. An important result due to J.Elworthy and A.Tromba states that a Fredholm structure on $M$ can be constructed from a Fredholm mapping $M \rightarrow H$ with zero index and also from certain smooth families of zero index Fredholm operators parametrized by the points of $M$ [11]. These facts were used in [15], [17] to construct Fredholm structures on loop groups. The following statement follows from the results of [15], [17].

Proposition 2. With any (complex) linear representation $\gamma$ of $U_{n}$ one can associate a Fredholm structure $F_{\gamma}$ on the group $L^{1} U_{n}$ of $H^{1}$-loops on $U_{n}$.

Here the loop group $L^{1} U_{n}$ is endowed with the usual $H^{1}$-norm [20]. It is easy to verify that with this norm it becomes a Hilbert Lie group.

Remark 4. Another proof of the same result may be derived from the results of [20] which yield in particular that the exponential map of the group of $H^{1}$ loops on a compact Lie group $G$ is a Fredholm map of index zero. Indeed, this local result enables one to obtain a Fredholm atlas on the loop group by merely taking the inverses of the exponential maps at every point composed with the natural identification of the tangent spaces with the Lie algebra of $L^{1} U_{n}$ (as always this can be done using differentials of right shifts on $L^{1} U_{n}$ which appear to have the form "identity + compact"). Details of this general argument will appear in a forthcoming paper by G. Khimshiashvili and G. Misiolek.

Remark 5. Actually Fredholm structures on loop groups come from several different sources. An interesting geometric way of constructing Fredholm structures on loop groups was suggested by D. Freed [12]. It is an intriguing open problem to investigate whether those "Fredholm" structures are compatible (concordant [11]) with the ones obtained from parameterizing the loop groups by families of Riemann-Hilbert problems as in [16], [17].

Such results can be interpreted in terms of Fredholm Grassmanians using the so-called Grassmanian models of loop groups [22]. As was explained in Section 2 , the realization of a loop as a coefficient of Riemann-Hilbert problem gives a natural mapping of an appropriate loop group $L U_{n}$ into the group $G L\left(\pi_{+}, K\right)$. In virtue of the above discussion (cf. also [22]) it is clear that by posing proper regularity conditions on a loop $f$ one can achieve that the rotation of subspace 
$H_{+}$by the operator of multiplication by $f$ gives a subspace in one of Grassmanians $G r^{s}$. In this way one obtains a natural mapping of $L G$ into $G r^{s}$ which is called the Grassmanian model (or embedding) of a loop group [22].

Some properties of these models follow from the preceding discussion, others were established in [22], basically for the case of the restricted Grassmanian $G r_{r}(H)$. In particular it is well known that the group of continuously differentiable loops can be embedded in $G r_{r}$ [22]. In general it is an interesting and difficult analytical problem to find exact regularity conditions which guarantee that the corresponding loop group can be realized in $G r^{s}$ (see examples presented in [22], Ch.7).

We avoid discussion of this problem by concentrating our attention on the group of smooth (infinitely differentiable) loops $L_{\infty} U_{n}$ which is the smallest of interesting groups of that kind. Its image under the above embedding is called the smooth (Fredholm) Grassmanian $G r_{\infty}$. It is easy to check that it lies in each Fredholm Grassmanian $G r^{s}$. A more interesting circumstance is that it is homotopy equivalent to each of them [22] so it captures important global properties of these Grassmanians.

Now one can transport various structures from $L_{\infty} U_{n}$ to $G r_{\infty}$. In particular it is evident that $L_{\infty} U_{n}$ can be endowed with Fredholm structures which are just the restrictions of the Fredholm structures on $H^{1}$-loops provided by Proposition 2 so we obtain the same conclusion for the smooth Grassmanian.

Proposition 3. With each linear (finite dimensional) representation of $U_{n}$

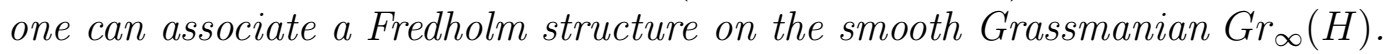

Of course one may ask whether it is possible to extend these structures to ambient Grassmanians $G r^{s}$ but this problem involves some delicate analytic issues which will be discussed elsewhere.

As an example of perspectives suggested by these results let us formulate another natural problem. From the mentioned result of Elworthy and Tromba and Proposition 2 it follows that there exists an index zero Fredholm mapping of the loop group $L^{1} U_{n}$ in Hilbert space. It would be interesting and instructive to find an explicit construction of such a mapping. The same problem can be formulated for all compact Lie groups. It would be also interesting to find such a mapping from the smooth Grassmanian $G r_{\infty}(H)$ in its model space.

Also, it is well known that for a Fredholm manifold $M$ one can define its characteristic classes $c h_{k}(M) \in H^{2 k}(M, \mathbf{Z})$. A natural and important problem is to identify these classes in the cohomology of $M$. In our setting this problem permits a particularly nice formulation.

As was already mentioned, the smooth Grassmanian has the same homotopy type as Fredholm Grassmanians $G r^{s}(H)$ so their cohomology rings are isomorphic and the structure of these rings is well-known [10]. It is also well known (see [10], [12]) that any Fredholm Hilbert manifold has well-defined Chern classes $c h_{j}$ which are classes in the even-dimensional cohomology of this manifold. Combining these two observations we conclude that a Fredholm structure on the smooth Grassmanian defines certain classes in $H^{2 j}\left(G r^{s}(H)\right)$. Thus we come to 
the problem of computing these classes for the structures $F_{\gamma}$ described above. Some results in this direction were obtained in [12], [13]. It is remarkable that such Chern classes can be represented by some differential forms defined using traces of appropriate products of operators from Schatten classes [13], which indicates an intriguing analogy with the non-commutative geometry of A.Connes $[7]$.

It seems worthy of noting that some properties of the so-called partial indices [21], [1] of Riemann-Hilbert problems can be also formulated in the language of Fredholm structures. As is well known (see [3]) the collections of matrix functions with the fixed partial indices, usually called Birkhoff strata [3], [22], define an interesting stratification of the loop group. Using the Grassmanian model of the loop group and Riemann-Hilbert problems described above, one obtains the corresponding strata in the smooth Grassmanian $G r_{\infty}$ and restricted Grassmanian $G r_{r}$ (cf. [22]). Using the known properties of partial indices [2], one can show that Birkhoff strata are complex analytic submanifolds of the finite codimension in $G r_{r}$ [8]. Combining this observation with Theorem 2 one becomes able to show that Birkhoff strata can be also treated in the context of Fredholm structures.

Proposition 4. The Birkhoff strata are Fredholm submanifolds of $G r_{\infty}(H)$ and, in virtue of the above discussion, each of them has a well defined fundamental class in the even-codimensional cohomology of $G r_{r}(H)$.

Some computations of the fundamental classes of Birkhoff strata can be found in $[8]$.

Using the same approach as above for loop groups and Fredholm Grassmanians associated with compact Lie groups [22] one can generalize Proposition 2 in this context. The formulation which we present follows from the results of [17] which in turn are based on the Fredholm theory for Riemann-Hilbert problems developed in [15], [17]. The existence follows from the Fredholmness of the corresponding linear conjugation problem for $G[16]$. Recall that for any compact Lie group one can naturally define the smooth Grassmanian $G r_{\infty}^{G}$ lying in $G r_{r}^{G}(H)$.

Proposition 5. For each linear representation $\gamma$ of a compact Lie group $G$, the smooth Grassmanian $G r_{\infty}^{G}(H)$ has a canonical Fredholm structure $F_{\gamma}$ induced by $\gamma$.

As was already mentioned for any Fredholm structure on a complex Banach manifold one can define its Chern classes [12], so we become able to introduce some global topological invariants of such Grassmanians.

Corollary 3. For each even $k$, there exists a canonical cohomology class in $H^{2 k}\left(G r_{\infty}^{G}(H)\right)$ which can be defined as the Chern class of the canonical Fredholm structure $F_{\gamma}$. 
It is evident that one can now formulate a number of natural questions related to such Fredholm structures.

In the conclusion we would like to express a hope that the approach described in this paper can lead to new insights about global properties of RiemannHilbert problems and geometric objects naturally associated with them.

\section{REFERENCES}

1. B. Bojarski, On the stability of Hilbert problem for holomorphic vector. Soobshch. Akad. Nauk Gruz. SSR 21(1958), 391-398.

2. B. Bojarski, The abstract linear conjugation problem and Fredholm pairs of subspaces. Differential and integral equations. Boundary value problems. Collection of articles dedicated to the memory of I. N. Vekua (eds. R. A. Kordzadze, G. F. Mandzhavidze), 45-60, Tbilisi University Press, Tbilisi, 1979.

3. B. Bojarski, Some analytical and geometrical aspects of the Riemann-Hilbert transmission problem. Complex Analysis. Methods, trends, applications ((ed. V. Tutschke), 97-110, Akademie-Verlag, Berlin, 1983.

4. B. Bojarski, The geometry of the Riemann-Hilbert problem. Contemp. Math. 242 (1999), 25-33.

5. B. Bojarski, The geometry of Riemann-Hilbert problem II. Boundary value problems and integral equations, 41-48, World Scientific, Singapore, 2000.

6. B. Booss-Bavnbek and K. Wojciechowski, Elliptic boundary problems for Dirac operators. Birkhäuser, Boston, 1993.

7. A. Connes, Non-commutative differential geometry. Academic Press, London, 1994.

8. S. Disney, The exponents of loops on a general complex linear group. Topology 12(1973), 297-315.

9. J. Eells, A setting for global analysis. Bull. Amer. Math. Soc. 72(1966), 751-802.

10. J. Eells, Fredholm structures. Proc. Sympos. Pure Math. 18(1970), 62-85.

11. J. Elworthy and A. Tromba, Differential structures and Fredholm maps on Banach manifolds. Proc. Sympos. Pure Math. 15(1970), 45-94.

12. D. Freed, The geometry of loop goups. J. Differential Geom. 28(1988), 223-276.

13. D. Freed, An index theorem for families of Fredholm operators parametrized by a group. Topology 27(1988), 279-300.

14. T. Kato, Perturbation theory of linear operators. Springer, Berlin, 1966.

15. G. N. Khimshiashvili, On the topology of the generalized Riemann-Hilbert problem. Soobshch. Akad. Nauk Gruz. SSR 135(1989), 241-244.

16. G. N. Khimshiashvili, On the Riemann-Hilbert problem for a compact Lie group. Dokl. Akad. Nauk SSSR 310(1990), 1055-1058.

17. G. Khimshiashvili, Lie groups and transmission problems on Riemann surfaces. Contemp. Math. 131(1992), 164-178.

18. G. Khimshiashvili, Elliptic transmission problems over $C^{*}$-algebras. Georgian Math. J. 5(1998), 511-521.

19. N. KuIPER, The homotopy type of the unitary group of Hilbert space. Topology $\mathbf{3}(1965)$, $19-30$.

20. G. Misiolek, The exponential map on a free loop space is Fredholm. Geom. Funct. Anal. 7(1997), 1-17. 
21. N. I. Muskhelishvili, Singular integral equations. (Russian) Nauka, Moscow, 1967; English translation from the 1st Russian edition: P.Noordhoff, Groningen, 1946.

22. A. Pressley and G. Segal, Loop groups. Clarendon Press, Oxford, 1986.

23. B. Simon, Trace ideals and their applications. Cambridge Univ. Press, Cambridge, 1979.

24. K. Wojciechowski, Spectral flow and the general linear conjugation problem. Simon Stevin Univ. J. 59(1985), 59-91.

(Received 10.09.2001)

Authors' addresses:

B. Bojarski

Institute of Mathematics

Polish Academy of Sciences

8, Sniadeckich St., Warsaw

Poland

E-mail: bojarski@impan.gov.pl

G. Khimshiashvili

A.Razmadze Mathematical Institute

Georgian Academy of Sciences

1, M. Aleksidze St., Tbilisi 380093

Georgia

E-mail: khimsh@rmi.acnet.ge 